\title{
Beam Instability Studies for the SSC
}

\author{
W. Chou \\ Superconducting Super Collider Laboratory* \\ 2550 Beckleymeade Avenue \\ Dallas, Texas 75237
}

September 1994

\footnotetext{
* Operated by the Universities Research Association, Inc., for the U.S. Department of Energy under Contract No. DE-AC35-89ER40486.
} 



\section{DISCLAIMER}

This report was prepared as an account of work sponsored by an agency of the United States Government. Neither the United States Government nor any agency thereof, nor any of their employees, make any warranty, express or implied, or assumes any legal liability or responsibility for the accuracy, completeness, or usefulness of any information, apparatus, product, or process disclosed, or represents that its use would not infringe privately owned rights. Reference herein to any specific commercial product, process, or service by trade name, trademark, manufacturer, or otherwise does not necessarily constitute or imply its endorsement, recommendation, or favoring by the United States Government or any agency thereof. The views and opinions of authors expressed herein do not necessarily state or reflect those of the United States Government or any agency thereof. 


\section{DISCLAIMER}

Portions of this document may be illegible in electronic image products. Images are produced from the best available original document. 


\title{
Beam Instability Studies for the SSC
}

\author{
W. Chou
}

\begin{abstract}
Beam instability studies for the Superconducting Super Collider (SSC) during the period 1989-1993 are briefly reviewed in this paper. Various topics are covered: single bunch and multi-bunch, single beam and beam-beam, parasitic heating and active feedback, etc. Although the SSC will not be built, many of the results obtained from these studies remain as useful references to the accelerator community.
\end{abstract}




\subsection{INTRODUCTION}

Studies on the collective beam instability problems for the Superconducting Super Collider (SSC) started in the early 1980s. A set of preliminary results were included in the SSC Conceptual Design, ${ }^{1}$ which was published in 1986. Since the establishment of the SSC Laboratory in 1989, these studies have been further pursued and numerous new results have been obtained. In this paper we will briefly review these results, including:

- Impedance budget and single bunch instability

- Higher-order modes and coupled bunch instability

- Resistive wall instability

- Feedback systems

- Parasitic heating

- Beam-beam effects.

The SSC is basically a low beam current machine. The beam intensity is primarily limited by the cryogenic system for absorbing the synchrotron radiation power from the $20-\mathrm{TeV}$ proton beams. Generally speaking, therefore, collective effects-such as single bunch instability, parasitic heating, and beam-beam interactions-do not present a threat to machine operations at normal beam intensity. However, there is an exception. That is the coupled bunch instability, which may become a real concern. This is because the number of bunches is enormous (about 17000 per beam, which is several orders of magnitude higher than in any existing storage rings), and because the required transverse emittance is extremely small (1 $\pi \mathrm{mm}$-mrad, normalized, which is just a fraction of that in any proton machines). These unique features of the SSC call special attention to the multi-bunch phenomena.

\subsection{IMPEDANCE BUDGET AND SINGLE-BUNCH INSTABILITY \\ 2.1 Impedance Budget of the Baseline Design}

In order to establish the impedance budget, each component in the vacuum system, rf system, diagnostic system, and injection/extraction systems has been carefully analyzed. Computer models for each component have been built, simulations have been carried out, and measurements for some critical components (e.g., the bellows and the liner) have been performed.

Two groups of simulation codes have been put in use. One is numerical, which is of either finite-difference type (such as the TBCI and MAFIA ${ }^{2}$ ) or finite-element type (such as the $\mathrm{HFSS}^{3}$ ). Another is based on an analytical model and is called the bperm (Boundary Perturbation Method), which was developed at the SSC. ${ }^{4}$ Benchmark tests for comparison 
purposes have been done, and the results for a given geometry obtained from different codes are in general agreement. As an example, Figure 1 shows the comparison of the wake potentials calculated by bperm and MAFIA for a tapered transition between two beam pipes with different cross sections.

The impedance budget is listed in Table 1 , where $Z_{\|} / n$ is the longitudinal impedance and $\mathrm{Z}_{\perp}$ the transverse one.

There are several remarks about this budget table:

1. Every effort has been made to make the beam pipe as smooth as possible: the bellows are shielded; the valves have rf fingers; the vacuum pump ports are screened; the transitions between two pipes of different sizes are tapered; and the ceramic pipes in the kicker sections are coated with thin metallic layers.

2. The 6000 bellows, even shielded, are a major contributor to the impedance. It is, therefore, worthwhile trying several different rf shield designs and comparing their impedance. The computer model of the baseline design (old design) is shown in Figure 2. The new design, which is similar to the design at the Large ElectronPositron (LEP) collider at CERN, is shown in Figure 3. Table 2 lists the comparison of the impedance of the two designs. It is a factor of four in difference. The significant reduction of the impedance of the new design comes from two facts: (1) The gap size is reduced from $3.4 \mathrm{~mm}$ to $2.5 \mathrm{~mm}$; (2) The right-hand-side of the gap is changed from an abrupt drop to a taper. Furthermore, in order to have a realistic estimate of the impedance, one needs to take into account the lateral misalignment during the installation, which would inevitably increase the impedance. The specification of the maximum lateral offset is $2.8 \mathrm{~mm}$. Assuming a uniform distribution in misalignment, the resulting increase in impedance is listed in Table 2.

3. The flange gap must be vacuum sealed. To reduce its impedance, it also needs to be rf sealed. There are several possible solutions to this problem. One is to use a separate rf finger to shield the gap, as the LEP did. Another is to use a thick copper coating on the flange surface that would serve both vacuum and rf sealing, as suggested by Helicoflex Company. A third solution is to use a movable rf finger, which would shield the bellows and flange gap simultaneously, as proposed for the Main Injector at Fermilab.

4. This budget table includes the components in both the cold region and warm region (i.e., the west utility, the east utility, and the interaction region). 

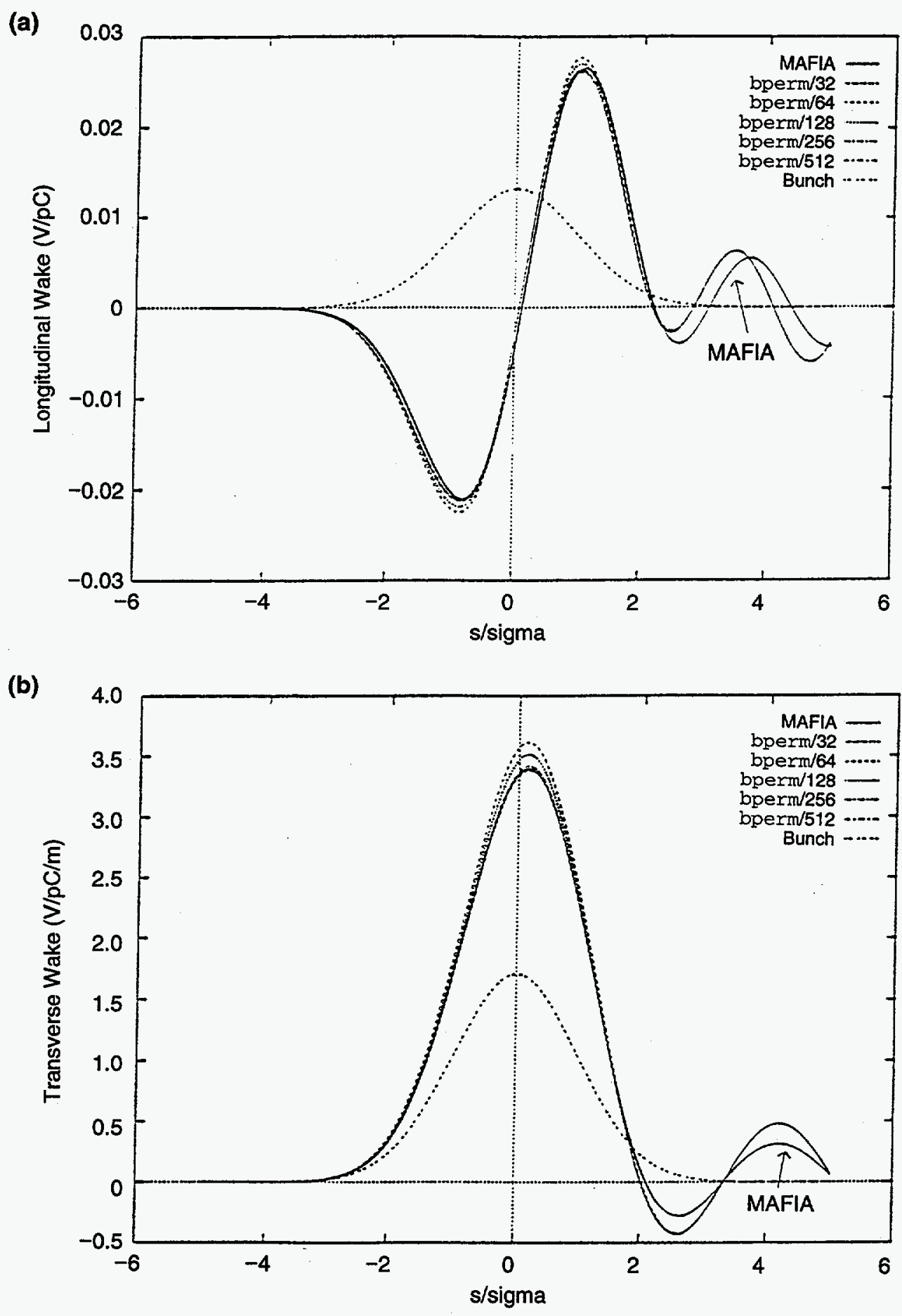

Figure 1. Comparison of the Longitudinal (a) and Transverse (b) Wake Potentials Calculated by bperm and MAFIA for a Tapered Structure. 
Table 1. Impedance Budget (per ring).

\begin{tabular}{|l|c|c|c|}
\hline \multicolumn{1}{|c|}{ Component } & Number & \multicolumn{2}{c|}{ Impedance } \\
\hline & & $\mathrm{Z}_{\|} / \mathrm{n}(\Omega)$ & $\mathrm{Z}_{\perp}(\mathrm{M} \Omega / \mathrm{m})$ \\
\hline Rf cavity (HOM) & $8 \times 5$-cell & 0.036 & 0.016 \\
Transition (tapered) & 4 & 0.004 & 0.003 \\
Bellows (shielded) & 6000 & 0.12 & 10 \\
BPM (15 cm, 55 ${ }^{\circ}$ ) & 968 & 0.05 & 4.6 \\
Weldment & 12000 & 0.002 & 0.2 \\
Valve (shielded) & 128 & $1 \mathrm{E}-4$ & 0.01 \\
Pump port (screened) & 650 & 0.02 & 2 \\
Flange gap & 12000 & $\mathrm{TBD}$ & $\mathrm{TBD}$ \\
Resistive wall & & 0.02 & 1.7 \\
Scrapers & & $1.8 \mathrm{E}-4$ & 0.02 \\
Collimators & & $2.6 \mathrm{E}-4$ & 0.08 \\
Injection Lambertson (laminated) & & $1.5 \mathrm{E}-3$ & 1.4 \\
Abort Lambertson (solid iron) & & - & - \\
Injection kicker & & 0.06 & 2.0 \\
Abort kicker & & 0.2 & 4.7 \\
Joint to Lambertson & & $\mathrm{TBD}$ & $\mathrm{TBD}$ \\
Conical section near IP & & - & - \\
\hline Total & & 0.51 & 27 \\
\hline Impedance budget = Total $\times 2$ & & 1.0 & 54 \\
\hline Instability threshold: & & 4.0 & 270 \\
\multicolumn{1}{|c|}{ At 2 TeV } & & 16 & 1200 \\
\hline
\end{tabular}




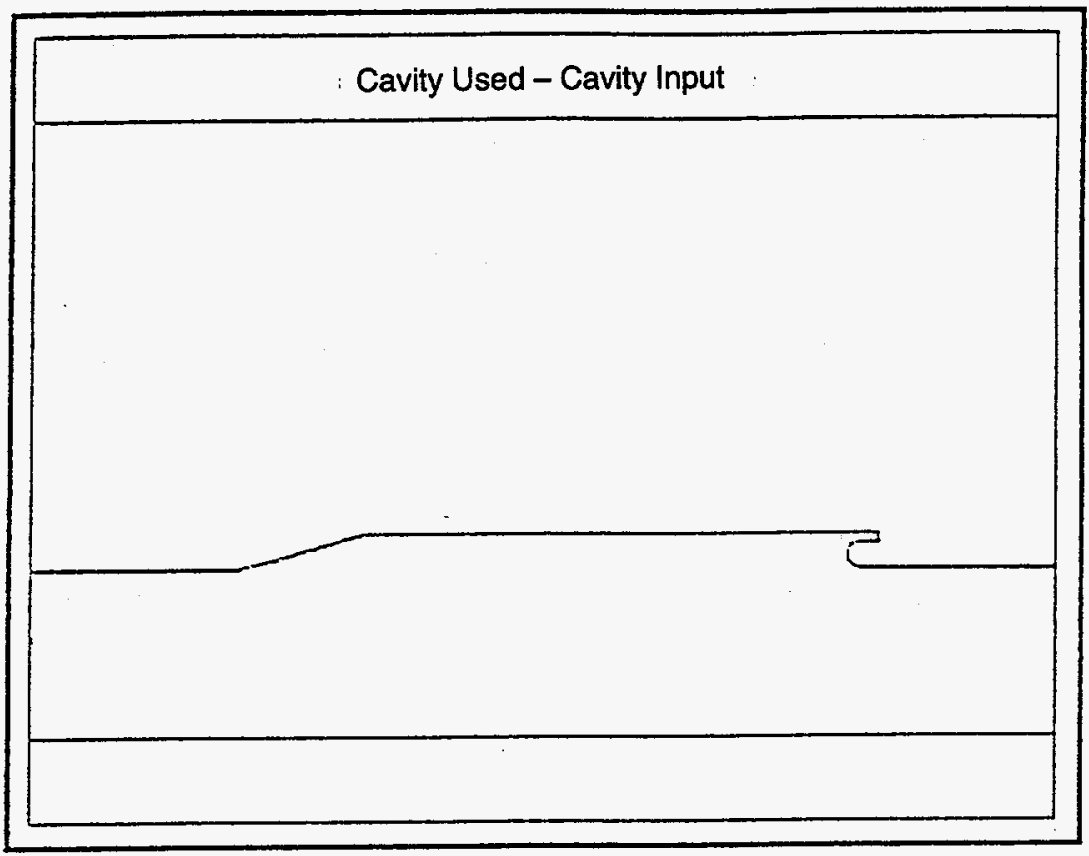

Figure 2. Computer Model of the Baseline Design (Old Design) of the Rf Shields for the Bellows. The discontinuity gap size in the radial direction is $3.4 \mathrm{~mm}$.

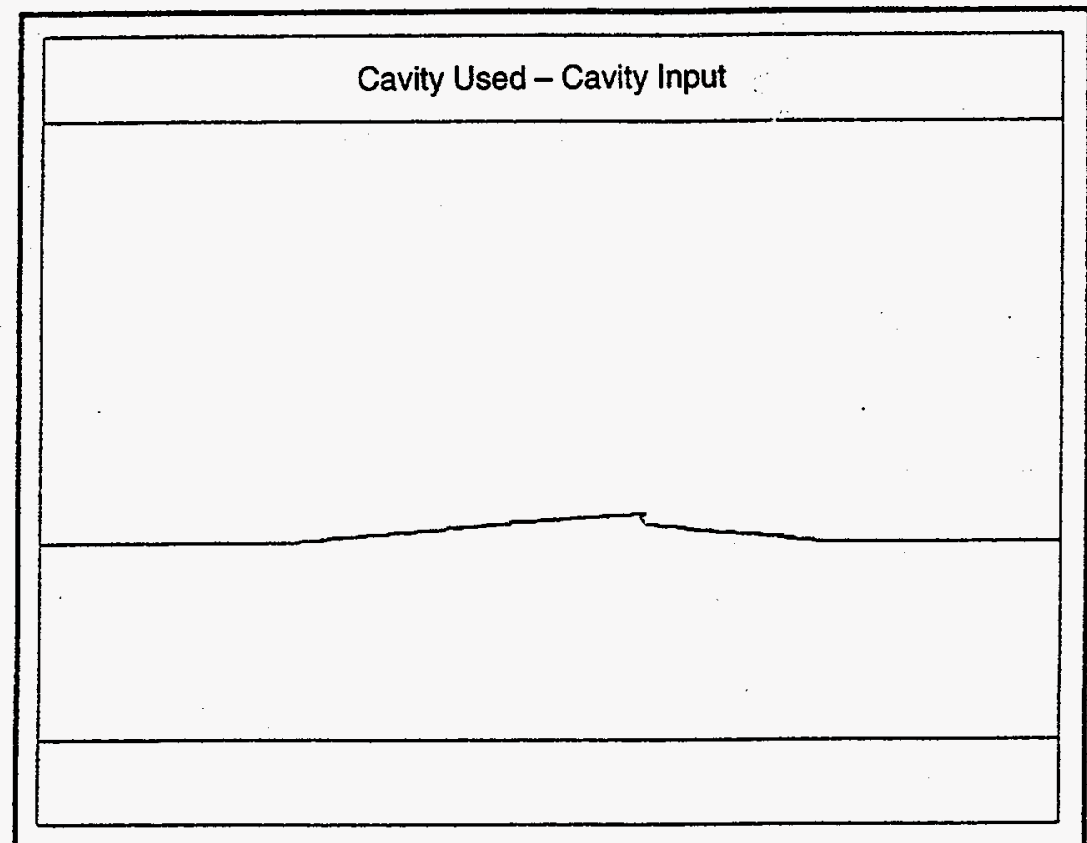

Figure 3. Computer Model of the New Design (LEP Design) of the Rf Shields for the Bellows. The discontinuity gap size in the radial direction is $2.5 \mathrm{~mm}$. 
Table 2. Comparison of Bellows (Shielded) Impedance.

\begin{tabular}{|l|c|c|}
\hline \multicolumn{1}{|c|}{ Case } & $\mathrm{Z}_{\|} / \mathrm{n}(\Omega)$ & $\mathrm{Z}_{\perp}(\mathrm{M} \Omega / \mathrm{m})$ \\
\hline A. Baseline (old design) & 0.12 & 10 \\
B. New design (LEP design) & 0.03 & 2.5 \\
$\quad$ No misalignment & & \\
C. New design & 0.06 & 6.5 \\
Max lateral offset 2.8 mm & & \\
Uniform distribution & & \\
\hline
\end{tabular}

5. In order to accommodate unforeseen sources in the impedance budget, the calculated total impedance is multiplied by a factor of two, which is then used in the safety margin estimate in Section 2.3.

\subsection{Impedance in the Presence of a Liner}

One plausible solution to the photodesorption problem is to install a perforated liner inside the beam pipe. This would increase the impedance in two ways:

1. The holes (or slots) on the surface of the liner would introduce additional impedance. At low frequencies (below the cutoff of the liner), the small holes (or slots) behave like a pure inductance. Their impedance can be estimated accurately. The results from the analytical models, code simulations, and wire measurements all agree with each other. ${ }^{5}$ It is also known that, for a given pumping area, short slots give less impedance than circular holes. At high frequencies (above the cutoff), however, the situation is more complex. Resonant peaks in the impedance spectrum are observed when the holes or slots are periodically placed. These peaks can be greatly suppressed when the periodicity is destroyed. It is thus concluded that randomly distributed short slots would be the best choice for the pattern of the perforation.

2. The installation of a liner would also reduce the inner radius (ID) of the pipe seen by the beam. Consequently, the transverse impedance of the components in the cold region would increase.

The baseline beam pipe ID is $33 \mathrm{~mm}$. Assuming the liner ID to be $25.3 \mathrm{~mm}$, hole diameter $2 \mathrm{~mm}$, and area coverage of the holes on the liner surface $4 \%$, the impedance increase is listed in Table 3. 
Table 3. Transverse Impedance With and Without Liner.

\begin{tabular}{|l|c|c|c|c|}
\hline \multicolumn{1}{|c|}{ Case } & $\begin{array}{c}Z_{\perp}^{(\text {liner })} \\
(\mathrm{M} \Omega / \mathrm{m})\end{array}$ & $\begin{array}{c}Z_{\perp}^{\text {(others) }} \\
(\mathrm{M} \Omega / \mathrm{m})\end{array}$ & $\begin{array}{c}Z_{\perp}^{\text {(total) }} \\
(\mathrm{M} \Omega / \mathrm{m})\end{array}$ & Safety Margin \\
\hline Baseline & - & 54 & 54 & 5 \\
With liner & 37 & 94 & 131 & 2 \\
\hline
\end{tabular}

\subsection{Single-Bunch Instability Threshold and Safety Margin}

The threshold impedance of the microwave instability (longitudinal) and mode-coupling instability (transverse) can be estimated by the following formulae:

$$
\begin{gathered}
\left|Z_{\|} / n\right|=\sqrt{\frac{\pi}{2}}\left(\frac{Z_{0}}{r_{\mathrm{p}}}\right)\left(\frac{\eta \gamma}{\beta}\right)\left(\frac{\sigma_{\mathrm{z}}}{N_{\mathrm{B}}}\right)\left(\frac{\sigma_{\mathrm{p}}}{p}\right)^{2} \\
\operatorname{Im} Z_{\perp}=2 \sqrt{\pi}\left(\frac{Z_{0}}{r_{\mathrm{p}}}\right)\left(\frac{\eta \gamma \nu_{\beta}}{\beta}\right)\left(\frac{1}{N_{\mathrm{B}}}\right)\left(\frac{\sigma_{\mathrm{p}}}{p}\right) .
\end{gathered}
$$

The transverse mode-coupling threshold obtained from Eq. (2) has been compared with that from the code BBI; ${ }^{6}$ they are in agreement. The ratio of the threshold impedance to the impedance budget, called the safety margin, is listed in Table 3.

It is seen that the introduction of a liner would significantly reduce the safety margin. Several measures could be taken to increase the safety margin:

- Enlarge the liner ID. This is the most effective way to reduce the transverse impedance. The scaling is

$$
Z_{\perp} \propto(\text { ID })^{-\alpha},
$$

where $\alpha=4$ for the liner, and $\alpha \approx 3$ for other components. In order to achieve a larger liner ID, one would have to increase the aperture of the quadrupoles and spool pieces from $4 \mathrm{~cm}$ to, say, $5 \mathrm{~cm}^{7}$

- Increase the longitudinal emittance $\epsilon_{\mathrm{L}}$. This would increase the impedance threshold:

$$
Z_{\perp}^{\text {(thresh) }} \propto \epsilon_{\mathrm{L}}^{1 / 2} .
$$

However, there are two concerns about a bigger $\epsilon_{\mathrm{L}}:(1)$ the rf bucket to bunch area ratio would be reduced; (2) the beam transfer from the High Energy Booster (HEB) to the Collider would be more difficult. 
- Increase the rf voltage at injection from 6.6 MV to $20 \mathrm{MV}$. This would also increase the impedance threshold:

$$
Z_{\perp}^{\text {(thresh) }} \propto V_{\mathrm{rf}}^{1 / 4} .
$$

One would have to reconsider the matching between the HEB and the Collider in this scenario.

\subsection{HIGHER-ORDER MODES AND COUPLED-BUNCH INSTABILITY}

The higher-order modes (HOM) of the rf cavities are the source of the coupled-bunch instability. The original choice of the rf cavity for the SSC is the 5-cell type used at the Positron-Electron Project (PEP) at Stanford. The HOM table is obtained from computer simulation using URMEL. ${ }^{8}$ The impedances of these HOMs are high and would cause coupled-bunch instabilities. The growth time is listed in Table 4.

Table 4. Coupled-Bunch Instability Growth Time.

\begin{tabular}{|l|c|c|c|}
\hline \multicolumn{1}{|c|}{ Direction } & Bunch Shape Mode & \multicolumn{2}{c|}{ Growth Time (s) } \\
\hline & & $2 \mathrm{TeV}$ & $20 \mathrm{TeV}$ \\
\hline Longitudinal & $m=1$ & 0.9 & 5.0 \\
& $m=2$ & 2.2 & 16 \\
\hline Transverse & $m=0$ & 8.6 & 91 \\
& $m=1$ & 16 & 150 \\
\hline
\end{tabular}

One successful method to cure the instability problem is to damp the HOMs. But it is difficult to do so in a multi-cell cavity. Therefore, a careful search has been carried out for choosing the best type of $\mathrm{rf}$ cavity for the SSC. ${ }^{9}$ Four types of rf cavities-multiple-cell and single-cell, superconducting (sc) and normal conducting (nc)-have been compared with respect to the specific needs of the SSC. The single-cell cavity is preferable to the multi-cell one because its HOMs are easier to damp. The sc cavity has a number of advantages over the nc one, but the mechanical complexity and operational reliability of the former may present a concern. The SSC rf committee, which was charged to make a recommendation, has endorsed the single-cell, sc cavity as the first choice for the SSC. With this type of cavity, the coupled bunch instability growth time would be more than an order of magnitude longer than that listed in Table 4.

The HOMs may also be generated if the beam pipes in the dipole and quadrupole sections have different cross sections. ${ }^{10}$ In this case, the large beam pipe can be regarded as parasitic cavities with certain eigenfrequencies and eigenmodes. These modes may get excited by a traversing charged particle bunch. When the mode frequencies are below the cutoff 
frequency of the small pipe, they will be trapped. If the quality factor of the trapped modes is high enough, they will stay there for a long time and will affect the behavior of the following bunches. The result could be a continuous beam emittance growth, as demonstrated in Reference 10. In order to avoid this effect, it was decided to use a beam pipe of uniform cross section throughout the entire cold region in the SSC.

\subsection{RESISTIVE WALL INSTABILITY}

The wall resistance of the beam tube may cause beam instability, which is usually of long correlation lengths and, therefore, shows no discrimination against the bunched beams from the coasting beams. The growth rate is

$$
\begin{aligned}
\frac{1}{\tau_{\mathrm{w}}} & =\frac{N_{\text {tot }} c r_{\mathrm{p}}}{2 \pi \gamma \nu_{\beta}} \frac{\operatorname{Re} Z_{\perp}}{R Z_{0}} \\
& =\frac{N_{\text {tot }} c r_{\mathrm{p}}}{2 \pi \gamma \nu_{\beta}} \frac{1}{b^{3}} \delta_{s},
\end{aligned}
$$

in which $\operatorname{Re} Z_{\perp}$ is the real part of the transverse impedance $Z_{\perp}$ of the wall, which is equal to

$$
Z_{\perp}=(1+i) \frac{Z_{0} R \delta_{s}}{b^{3}}
$$

The skin depth $\delta_{s}$ is a function of the wall conductivity $\sigma_{e}$ and frequency $\omega$ :

$$
\delta_{s}=\sqrt{\frac{2}{\mu \sigma_{e} \omega}} .
$$

It is seen that as the frequency is decreased, $\delta_{s}$ is increased as $\omega^{-1 / 2}$. So is the growth rate $1 / \tau_{\mathrm{w}}$. The frequency range of interest is $\omega=\left(n-\nu_{\beta}\right) \omega_{0}$, in which $n$ is an integer and $\omega_{0}=c / R$ is the angular revolution frequency, which equals $2 \pi \times 3.441 \mathrm{kHz}$ for the Collider. During machine operation, the fractional part of $\nu_{\beta}$ would be scanned in order to find the optimal working point. Therefore, the lowest frequency is taken to be $0.1 \omega_{0}$ in the estimates of the fastest growth time.

In the baseline design, the beam tube of the Collider is made of stainless steel, which is coated on its inner surface with a thin copper layer for low electrical resistivity. In this case, the thickness of the copper layer $\Delta$ is smaller than the skin depth $\delta_{s}$ in the frequency range mentioned above, and Eq. (6) needs to be modified. ${ }^{11}$ With some approximations, the modified equation can be written as

$$
\frac{1}{\tau_{\mathrm{w}}}=\frac{N_{\mathrm{tot}} c r_{\mathrm{p}}}{2 \pi \gamma \nu_{\beta}} \frac{1}{b^{3}} \frac{\delta_{s}^{2}}{\Delta}
$$


Plugging in Eq. (8) and expressing the instability in terms of the growth time, one has

$$
\tau_{\mathrm{w}}=\left(\frac{2 \pi \gamma \nu_{\beta} b^{3}}{N_{\text {tot }} c r_{\mathrm{p}}} \frac{\mu \omega}{2}\right) \sigma_{e} \Delta
$$

In other words, the growth time is proportional to the product of $\sigma_{e} \Delta$. The specification of the copper layer is

$$
\sigma_{e} \Delta \geq 2 \times 10^{5} \quad 1 / \Omega
$$

in which $\sigma_{e}$ is the conductivity of the copper layer at $4 \mathrm{~K}$ and $0.66 \mathrm{~T}$. The nominal values are $\sigma_{e}=2 \times 10^{9} \Omega^{-1} \mathrm{~m}^{-1}$ (corresponding to $\mathrm{RRR}=30$ ), $\Delta=0.1 \mathrm{~mm}$. For a beam tube ID of $33 \mathrm{~mm}$ and at $\omega=0.1 \omega_{0}$, these parameters would give a wall impedance of $4300 \mathrm{M} \Omega / \mathrm{m}$ in the cold region. The wall impedance in the warm regions also needs to be considered. Table 5 is a complete list of the wall impedance in the SSC.

Table 5. Resistive Wall Impedance.

\begin{tabular}{|l|c|c|}
\hline \multicolumn{1}{|c|}{ Component } & \multicolumn{2}{c|}{$\mathrm{Z}_{\perp}(\mathrm{M} \Omega / \mathrm{m})$} \\
\hline Cold region beam pipe & $2 \mathrm{TeV}$ & $20 \mathrm{TeV}$ \\
Warm region beam pipe (stainless steel) & 4300 & 4300 \\
Graphite shadows: & 1300 & 1300 \\
$\quad$ & & \\
$\quad$ Upstream to abort Lambertson & 7.1 & 7.1 \\
$\quad$ Upstream to collimator & 10 & 323 \\
Scrapers (copper) & 1.4 & 46 \\
Collimators (stainless steel) & 7.7 & 250 \\
Abort Lambertson (solid iron): & & \\
$\quad$ Symmetric & 22 & 22 \\
$\quad$ Asymmetric & 4.6 & 4.6 \\
\hline Total & 5700 & 6300 \\
\hline
\end{tabular}

The total wall impedance would give an instability growth time of $25 \mathrm{~ms}$, or 88 turns, during the approximately one hour injection period $(2 \mathrm{TeV})$. This would have to be damped by an active feedback system, as will be discussed in Section 5 . At full energy $(20 \mathrm{TeV})$, the growth rate would be slower due to the beam rigidity. In addition, the large betatron tune spread resulting from beam-beam collisions may provide significant Landau damping.

There is an alternative to the copper-coated stainless steel beam pipe: an aluminum alloy beam tube. ${ }^{12}$ There are several important reasons for considering this option, e.g., saving the high cost of the copper coating, solving the vacuum problem without a liner, 
and avoiding the adhesion problem in a bi-layer tube. As far as the beam instability is concerned, the issue is the surface resistance of the aluminum tube. Table 6 is a comparison of the surface resistance between the copper-coated stainless steel and the aluminum alloy 7039-T61, which is of high strength and high resistivity. The quantity $\sigma_{e} \Delta$ is comparable in the two cases. Therefore, the use of an aluminum beam pipe should not affect the resistive wall instability growth time.

Table 6. Comparison of Surface Resistance.

\begin{tabular}{|l|c|c|c|c|}
\hline \multicolumn{1}{|c|}{ Material } & $\begin{array}{c}\sigma_{e} \\
\left(\Omega^{-1} \mathrm{~m}^{-1}\right)\end{array}$ & $\begin{array}{c}\delta^{\dagger} \\
(\mathrm{mm})\end{array}$ & $\begin{array}{c}\Delta \\
(\mathrm{mm})\end{array}$ & $\begin{array}{c}\sigma_{e} \Delta \\
\left(\Omega^{-1}\right)\end{array}$ \\
\hline Cu layer (RRR = 30) & $1.8 \times 10^{9}$ & 0.6 & 0.1 & $1.8 \times 10^{5}$ \\
Al 7039-T61 tube & $5.6 \times 10^{7}$ & 3.6 & 3 & $1.7 \times 10^{5}$ \\
\hline
\end{tabular}

${ }^{\dagger}$ At $\omega=0.1 \omega_{0}$.

\subsection{FEEDBACK SYSTEMS}

An effective way to cure beam instabilities is to build active feedback systems. In this paper, we will content ourselves with the discussion of transverse feedback systems. These systems serve four different purposes:

\section{Correction of the injection errors}

At injection there are errors in the beam position, angle, energy, and phase that uncorrected can lead to beam emittance dilution. Because these errors are relatively large compared with other types of perturbations described below, the feedback system must have enough power to quickly kick the beam back to the desired orbit before any significant decoherence occurs.

2. Damping of the resistive wall instability

This was discussed in Section 4 above. Because it is a fast beam blowup in the transverse planes, a feedback system with a large gain is needed.

3. Damping of the coupled bunch instability driven by the HOM of rf cavities

As discussed in Section 3, if the HOMs of rf cavities are not properly damped and/or staggering-tuned, they may drive the beam unstable. The feedback system needs a wide bandwidth to cure this type of instability.

4. Control of possible emittance growth from the above and/or other mechanisms (e.g., ground motion, coolant flow, power supply ripples, etc.)

Because of the long memory of the proton beam (the radiation damping time in the SSC is about $13 \mathrm{~h}$ ), any external perturbation will be remembered by the beam and may lead to eventual emittance growth. A feedback system that keeps the coherent 
motion of the beam below a certain allowable amplitude can effectively reduce the emittance growth rate. This feedback system must have a very low noise level.

It is seen that each purpose imposes different requirements on the feedback systems. This, of course, does not mean that a total of four systems would be required. One system may well serve several different purposes. Table 7 lists three systems and their main parameters.

Table 7. Power, Bandwidth, Gain, and Noise Level Specifications.

\begin{tabular}{|l|c|c|c|}
\hline Feedback System & A & B & C \\
\hline Purpose & Injection errors & $\begin{array}{l}\text { Resistive wall instability } \\
\text { emittance control }\end{array}$ & Multi-bunch instability \\
Gain & 0.04 & 0.1 & 0.02 \\
Damping time & 50 turns & 20 turns & 100 turns \\
Max. correction & $\pm 2 \mathrm{~mm}$ & $\pm 100 \mu \mathrm{m}$ & $\pm 40 \mu \mathrm{m}$ \\
Kick angle & $0.27 \mu \mathrm{rad}$ & $0.04 \mu \mathrm{rad}$ & $0.003 \mu \mathrm{rad}$ \\
Kicker length & $4 \mathrm{~m}$ & $4 \mathrm{~m}$ & $4 \mathrm{~m}$ \\
Kicker voltage & $1 \mathrm{kV}$ & $150 \mathrm{~V}$ & $150 \mathrm{~V}$ \\
Kicker power & $40 \mathrm{~kW}$ & $0.9 \mathrm{~kW}$ & $0.9 \mathrm{~kW}$ \\
Bandwidth & $500 \mathrm{kHz}$ & $500 \mathrm{kHz}$ & $\geq 30 \mathrm{MHz}$ \\
Noise level & - & $\leq 2 \mu \mathrm{m}$ & $\leq 1 \mu \mathrm{m}$ \\
Resolution limit & $0.02 \mu \mathrm{m}$ & $0.02 \mu \mathrm{m}$ & $0.16 \mu \mathrm{m}$ \\
\hline
\end{tabular}

For proton storage rings, one would have to specify the noise level in the feedback systems in order to keep the emittance growth below a certain allowable level. The emittance growth rate due to the noise has been worked out: ${ }^{13}$

$$
\frac{1}{\tau_{\text {noise }}}=\frac{1}{\epsilon_{0}} \frac{f_{0} g^{2} x_{\mathrm{N}}^{2}}{2 \beta_{\mathrm{x}}}\left(\frac{4 \sqrt{2} \sigma_{\nu}}{g}\right)^{2}=0.64 f_{0}\left(\frac{x_{\mathrm{N}}}{\sigma_{\beta}}\right)^{2} \Delta \nu^{2} .
$$

On the other hand, one can also estimate the theoretical limit of the pickup resolution that comes from the thermal and electronic noises:

$$
\Delta x=\frac{2 d}{I_{\mathrm{av}} \sin \left(\frac{\omega \ell}{c}\right)} \sqrt{\frac{k_{\mathrm{B}} T \cdot \Delta f \cdot 10^{N F / 10}}{Z}} .
$$

These formulae are used to specify the noise levels and estimate the resolution limits listed in Table 7 . In designing a feedback system, the quantity $\Delta x$ must be smaller than $x_{\mathrm{N}}$, which is determined by a specified allowable emittance growth rate $1 / \tau_{\text {noise }}$. 


\subsection{PARASITIC HEATING}

The parasitic heating attributed to the surface resistance $R_{\mathrm{s}}$ of the beam pipe can be calculated by

$$
P=k \frac{I_{\mathrm{av}}^{2}}{M f_{0}}
$$

in which the loss factor is

$$
k=\frac{c^{2}}{2 \pi} \int_{-\infty}^{\infty} \tilde{\lambda}^{2}(\omega) R_{\mathrm{wall}}(\omega) d \omega,
$$

where the wall resistance $R_{\text {wall }}$ is related to the surface resistance by

$$
R_{\text {wall }}=\frac{R}{b} R_{\mathrm{s}}
$$

In order not to exceed the heat load budget set for the cryogenic system (which is $1 \mathrm{~kW}$ per ring for the parasitic heating), the surface resistance must be kept below a certain level. Because the parasitic heating is a high-frequency phenomenon (the SSC bunch spectrum is centered at about $1 \mathrm{GHz}$ ), one would have to consider the anomalous skin effect. But the actual situation is more complicated. To get a complete picture, one should consider the co-existence of the following three extreme conditions:

- Low temperature (4 K).

The wall resistance of many materials at low temperature is lower than that at room temperature. This is usually described by a quantity called the residual resistance ratio (RRR). For pure metals (e.g., copper and aluminum), RRR can be hundreds or even thousands at helium temperature, depending on their purity. For alloys (e.g., stainless steel and aluminum alloy), RRR is in the order of unity. But this quantity is meaningful only at low frequencies and low magnetic field.

- High magnetic field (6.6 T).

In a magnetic field, one would have to consider the magnetoresistance. This can be studied using a Kohler plot, which describes the dependence of the resistance on the magnetic field. At 6.6 T, the RRR values could be an order of magnitude lower than that at zero field.

- High frequency ( $1 \mathrm{GHz}$ and above).

When the frequency is high enough such that the mean free path of electrons becomes larger than the skin depth, the normal conduction theory based on electron collisions breaks down and the surface resistance becomes independent of the conductivity $\sigma_{e}$ of the material. This is called the anomalous skin effect. The surface resistance ratio $R_{\mathrm{s}}(300 \mathrm{~K}) / R_{\mathrm{s}}(4 \mathrm{~K})$ of copper at high frequencies is significantly lower than the dc 
value. The preliminary results measured by the Los Alamos National Laboratory are listed in Table 8.

Therefore, in order to have an accurate estimate of the parasitic heating, one would have to measure the surface resistance of the beam pipe in the presence of all three conditions. This work is not completed.

Table 8. Surface Resistance Ratio of a Copper-Plated Tube.

\begin{tabular}{|c|c|}
\hline Frequency $(\mathrm{GHz})$ & $R_{s}(300 \mathrm{~K}) / R_{s}(4 \mathrm{~K})$ \\
\hline $\mathrm{dc}$ & 107 \\
0.959 & 4 \\
1.865 & 3.2 \\
7 & 3.7 \\
\hline
\end{tabular}

\subsection{BEAM-BEAM EFFECTS}

There have been extensive studies on proton beam-beam interactions at the Tevatron (Fermilab) and Spp $\mathrm{S}$ (CERN). Their experiences are important. There are, however, three new features of the SSC with respect to the beam-beam effects: the gaps in the bunch train (which produce the so-called Pacman effect), the short bunch spacing (which leads to long-range beam-beam interactions), and the finite crossing angle (which gives rise to synchro-betatron resonances and reduces the luminosity).

\subsection{Strong Beam-beam Interactions}

These interactions can result in inelastic scattering and elastic scattering.

1. Inelastic scattering:

Particles are lost in this process. The loss rate is

$$
\frac{d N}{d t}=\mathcal{L} \sigma_{\text {inel }}
$$

which is $10^{8} \mathrm{~s}^{-1}$ per interaction point (IP) at the design luminosity $10^{33} \mathrm{~cm}^{-2} \mathrm{~s}^{-1}$. The corresponding beam-beam luminosity lifetime is $180 / N_{\text {IP }}$ hours.

2. Elastic scattering: ${ }^{14}$

This contributes to the emittance growth. The growth rate is

$$
\frac{d \epsilon}{d t}=\frac{N_{\mathrm{B}} f_{0}}{4 \pi \epsilon} \sigma_{\mathrm{el}} \sigma_{\theta}^{2}
$$

which is about $4.6 \times 10^{-17} \mathrm{~m}-\mathrm{rad} / \mathrm{s}$ per IP. 


\subsection{Electromagnetic Beam-beam Interactions}

There are two types of interactions: head-on and long-range (which are also called parasitic crossings). The characteristic quantity of head-on collisions is the beam-beam parameter $\xi$, which for a round beam is defined by

$$
\xi=\frac{r_{\mathrm{p}} N_{\mathrm{B}}}{4 \pi \epsilon_{\mathrm{N}}} .
$$

It equals 0.0009 at the SSC and is substantially smaller than that at the Tevatron and $\mathrm{S} p \bar{p} \mathrm{~S}(0.003-0.006)$. The strength of long-range interactions is mainly determined by the beam intensity and the beam separation at parasitic crossing points. The latter is about $7.5 \sigma_{\beta}$ for a crossing angle of $75 \mu \mathrm{rad}$.

1. Incoherent effects:

(a) Tune shift and tune spread:

The most significant beam-beam effect observed at the Tevatron and $S p \bar{p} S$ is the slow diffusion caused by high-order betatron resonances. It leads to particle loss, which in turn decreases the beam lifetime and creates background in detectors. The tune shift per IP due to head-on collisions is

$$
\Delta \nu_{\mathrm{HO}}=\xi\left(\frac{2 R_{\mathrm{re}}^{2}}{1+R_{\mathrm{re}}}\right),
$$

which is also the tune spread. For long-range interactions, the tune shift per IP is

$$
\Delta \nu_{\mathrm{LR}}=\frac{r_{\mathrm{p}} N_{\mathrm{B}} L^{*}}{\pi \gamma \beta^{*} \alpha^{2} S_{\mathrm{B}}} .
$$

The tune spread per IP is

$$
\delta \nu_{\mathrm{LR}}=\frac{3 r_{\mathrm{p}} n^{2} \epsilon_{\mathrm{N}} N_{\mathrm{B}} L^{*}}{2 \pi \gamma^{2} \beta^{* 2} \alpha^{4} S_{\mathrm{B}}} .
$$

In order to control the slow diffusion, it is required to keep the total tune spread (head-on + long-range + nonlinear magnetic field) within the tune budget, which is set to be 0.02 . The linear tune shift due to long-range tune spread may be compensated in principle by retuning quadrupoles. But the Pacman effect discussed below makes it difficult.

The nominal values at each low- $\beta^{*}$ IP are: $\Delta \nu_{\mathrm{HO}}=0.0009, \Delta \nu_{\mathrm{LR}}=0.0022$, $\delta \nu_{\mathrm{LR}}=0.0009$. Even with a total of four IPs (two low- $\beta^{*}$ and two medium- $\beta^{*}$ ), it should be possible to keep the total tune shift and tune spread within the tune budget. 
(b) Orbit distortion:

This is induced by long-range interactions and is given by

$$
\delta x=-\frac{4 N_{\mathrm{B}} r_{\mathrm{p}} L^{*}}{\alpha \gamma S_{\mathrm{B}}} \sqrt{\frac{\beta_{\mathrm{x}}}{\beta^{*}}} \frac{\sin \left(\pi \nu_{\beta}-\phi\right)}{\sin \left(\pi \nu_{\beta}\right)} .
$$

The calculated values are small compared with the beam size at the IPs (less than $10 \% \sigma_{\beta}$ ). Fine steering is desired near the IPs for orbit corrections.

2. Coherent effects:

Both head-on and long-range interactions can produce coherent beam-beam effects. The rigid dipole modes ( $\pi$-mode and $\sigma$-mode) and high-order multipole modes are studied by theoretical modeling and by computer simulations. The results are expressed in terms of the stability boundary in the $\left(\xi, \nu_{\beta}\right)$ space. There is enough room for the working area during normal SSC operations.

3. Pacman effect:

In the SSC bunch train there are seven injection gaps (1.7 $\mu$ s each) and one abort gap $(4.1 \mu \mathrm{s})$. Bunches near the edge of the gaps may miss collisions at some IP, thus experiencing an irregular collision sequence. These bunches will have different orbit distortion, different tune shift, and different tune spread from those with a normal collision sequence. This makes the orbit and tune correction difficult. But simulations show that there should be enough working area in the tune space to accommodate the additional tune shift and spread due to the Pacman effect.

4. Synchro-betatron resonance: ${ }^{15}$

The finite crossing angle will excite synchro-betatron resonances. Computer simulations show that this is not a serious problem at the SSC. This is because the three main parameters that determine the strength of the resonances are small at the SSC. They are: (a) the beam-beam parameter $\xi(0.0009)$, (b) the synchrotron tune $\nu_{s}(0.0012)$, and (c) the normalized crossing angle $\alpha \sigma_{z} / \sigma_{\beta}(0.45)$. With $\alpha=150 \mu \mathrm{rad}$, only the satellites of the resonances up to the order of six could be harmful to the beams. Between these resonances there is enough space for the working area.

\subsection{CONCLUSION}

Although the SSC will never be built, the results of beam instability studies during the past four years may still serve as useful references for future accelerators. Moreover, the new tools (e.g., the code bperm), new measurements (e.g., the surface resistance at low temperature, high frequencies, and high magnetic field), and new analysis (e.g., the trapped $\mathrm{rf}$ modes effect, the noise level specification in a feedback system, the aluminum 
alloy beam tube, and the liner impedance) that have been developed at the SSC will remain as valuable properties of the whole accelerator community. 



\section{REFERENCES}

1. SSC Conceptual Design, SSC-SR-2020, SSC Laboratory (1986).

2. MAFIA is a commercial code developed by $\mathrm{T}$. Weiland and his associates. TBCI is a subset of MAFIA.

3. HFSS is a commercial code developed by Hewlett-Packard Company.

4. T. Barts and W. Chou, A User's Guide for the Computer Code BPERM-A Boundary Perturbation Method for Wakepotential and Impedance Calculations, SSCL-MAN-0035, SSC Laboratory (1994).

5. W. Chou and T. Barts, "Impedance of a Perforated Liner and Its Impact on the SSC Collider," and the references therein, p. 3444, Proc. 1993 Particle Accelerator Conference, Washington, D.C., May 17-20, 1993; also see SSCL-Preprint-425 (1993).

6. BBI is a computer code written by B. Zotter and his associates at CERN.

7. H. T. Edwards, Study on Beam Tube Vacuum with Consideration of Synchrotron Light, Potential Liner Intercept, and Collider Quad/Spool Coil Diameter, SSCL-N-771, SSC Laboratory (1991).

8. URMEL is a computer code written by T. Weiland.

9. W. Chou, "Choice of the RF Cavity for the SSC Collider," p. 818, Proc. 1993 Particle Accelerator Conference, Washington, D.C., May 17-20, 1993; also see SSCL-Preprint-424 (1993).

10. W. Chou, "Impact of Cross-Sectional Changes in the Beam Tube on Beam Dynamics," p. 1710, Proc. 1991 Particle Accelerator Conference, San Francisco, May 6-9, 1991; also see SSCL-420 (1991).

11. J. D. Jackson, Surface Impedance of a Two-Layer Beam Tube and Resistive-Wall Contribution to Machine Impedance, SSC-N-110, SSC Laboratory (1986).

12. W. Chou, Feasibility Study of Aluminum Beam Tube for the Collider-An Option for No-Coating and No-Liner, SSCL-649, SSC Laboratory (1994).

13. W. Chou and J. Peterson, "Issues of the Transverse Feedback Systems Design at the SSC," p. 2281, Proc. 1993 Particle Accelerator Conference, Washington, D.C., May 17-20, 1993; also see SSCL-Preprint-414 (1993). W. Chou and J. Peterson, "The SSC Transverse Feedback Systems," p. 188, Proc. the 19th and 25th Workshops of the INFN Eloisatron Project, Erice, Italy, Nov 17-22, 1992, edited by W. A. Barletta and H. Leutz, published by World Scientific (1993); also see SSCL-623 (1993). 
14. W. Chou, S. Dutt, T. Garavaglia, and S. Kauffmann, "Emittance and Luminosity Evolution During Collisions in the SSC Collider," p. 3609, Proc. 1993 Particle Accelerator Conference, Washington, D.C., May 17-20, 1993; also see SSCL-Preprint-415 (1993).

15. W. Chou and A. Piwinski, "Synchro-Betatron Resonances Excited by the Beam-Beam Interaction at a Crossing Angle in the SSC," p. 134, Proc. of B Factories: The State of the Art in Accelerators, Detectors and Physics, SLAC, Stanford, California, April 6-10, 1992, SLAC-400 (1992); also see SSCL-Preprint-119 (1992). 


\section{Glossary}

$\begin{array}{ll}Z_{\|} / \mathrm{n} & \text { longitudinal impedance } \\ Z_{\perp} & \text { transverse impedance } \\ Z_{0} & \text { vacuum impedance } \\ \gamma & \text { relativistic energy of the particle } \\ \beta & \text { relativistic velocity } \\ \eta & \text { slip factor } \\ \mathrm{IP} & \text { interaction point } \\ \sigma_{\mathrm{z}} & \text { rms bunch length } \\ \sigma_{\mathrm{p}} / p & \text { rms relative momentum spread } \\ N_{\mathrm{B}} & \text { number of particles per bunch } \\ \nu_{\beta} & \text { betatron tune } \\ r_{\mathrm{p}} & \text { classical radius of proton } \\ \epsilon_{\mathrm{L}} & \text { rms longitudinal emittance } \\ V_{\mathrm{rf}} & \text { rf peak voltage } \\ \tau_{\mathrm{w}} & \text { resistive wall instability growth time } \\ N_{\text {tot }} & \text { total number of particles } \\ c & \text { velocity of light } \\ R & \text { average machine radius } \\ \delta_{s} & \text { skin depth } \\ b & \text { beam tube radius } \\ \mu & \text { vacuum permeability } \\ \sigma_{e} & \text { wall conductivity } \\ \omega & \text { angular frequency } \\ \omega_{0} & \text { angular revolution frequency } \\ \Delta & \text { coating layer thickness } \\ \mathrm{RRR} & \text { residual resistance ratio } \\ \mathrm{ID} & \text { inner radius } \\ g & \text { gain of a feedback system } \\ x_{\mathrm{N}} & \text { noise level at the pickup } \\ \sigma_{\nu} & \text { rms tune spread } \\ \Delta \nu & \text { total tune spread } \\ \sigma_{\beta} & \text { rms beam size } \\ \beta_{\mathrm{X}} & \text { beta function } \\ \Delta x & \text { theoretical limit of pickup resolution } \\ & \end{array}$




\begin{tabular}{ll}
$k_{\mathrm{B}}$ & Boltzmann constant \\
$T$ & temperature in Kelvin \\
$\Delta f$ & bandwidth of a feedback system \\
$N F$ & noise factor (in dB) of a feedback system \\
$d$ & half distance between two pickup electrodes \\
$Z$ & characteristic impedance \\
$\ell$ & length of the electrodes \\
$I_{\mathrm{av}}$ & average beam current \\
$M$ & number of particle bunches \\
$f_{0}$ & revolution frequency \\
$k$ & loss factor \\
$\tilde{\lambda}(\omega)$ & bunch spectrum \\
$R_{\mathrm{wall}}$ & wall resistance \\
$R_{\mathrm{S}}$ & surface resistance \\
$\mathcal{L}$ & luminosity \\
$\epsilon$ & transverse emittance \\
$\epsilon_{\mathrm{N}}$ & normalized transverse emittance \\
$N_{\mathrm{IP}}$ & number of interaction points \\
$\sigma_{\mathrm{inel}}$ & inelastic cross section \\
$\sigma_{\mathrm{el}}$ & elastic cross section \\
$\sigma_{\theta}$ & rms value of $p p$ elastic scattering angle in the center of mass system \\
$\xi$ & beam-beam parameter \\
$\alpha$ & crossing angle \\
$R_{\mathrm{re}}$ & luminosity reduction factor due to the crossing angle \\
$L^{*}$ & effective interaction distance \\
$S_{\mathrm{B}}$ & bunch spacing \\
$\beta^{*}$ & $\beta$-function at the interaction point \\
$\nu_{s}$ & synchrotron tune \\
$\Delta \nu_{\mathrm{HO}}$ & head-on beam-beam tune shift \\
$\Delta \nu_{\mathrm{LR}}$ & long-range beam-beam tune shift \\
$\delta \nu_{\mathrm{LR}}$ & long-range beam-beam tune spread \\
$\delta x$ & orbit distortion \\
$\phi$ & phase advance \\
$n$ & Betatron oscillation amplitude in unit $\sigma_{\beta}$ \\
& \\
\hline &
\end{tabular}

